\title{
Bacterial colonisation of collagen-coated polypropylene vaginal mesh: are additional intraoperative sterility procedures useful?
}

\author{
Astrid Vollebregt • Annet Troelstra • \\ C. Huub van der Vaart
}

Received: 24 February 2009 / Accepted: 21 June 2009 / Published online: 2 September 2009

(C) The Author(s) 2009. This article is published with open access at Springerlink.com

\begin{abstract}
Introduction and hypothesis The use of vaginally implanted polypropylene meshes in the treatment of prolapse is becoming increasingly popular. We set out to detect how often bacterial colonisation of the mesh occurs and if the intraoperative sterility procedures that are applied matter.

Methods In 64 consecutive women, bacterial colonisation was compared between two intraoperative sterility procedures. Culture swabs of the core mesh were taken during surgery, and the mesh arms removed at the end of surgery were cultured separately.

Results Sixty-seven implants were cultured. In 56 (83.6\%) implants, a positive culture with vaginal bacteria was found with very low bacterial density $\left(<10^{3}\right.$ colony-forming units). No significant differences in bacterial species, density, clinical infection and erosion (two anterior and one posterior) were found between the two intraoperative sterility methods.

Conclusions Colonisation of vaginally implanted mesh occurs frequently but in low bacterial densities, irrespective of the intraoperative sterility procedure used.
\end{abstract}

\footnotetext{
A. Vollebregt $(\bowtie)$

Department of Obstetrics and Gynaecology, Spaarne Hospital, P.O. Box 770, 2130 AT Hoofddorp, The Netherlands

e-mail: a.vollebregt@umcutrecht.nl
}

\section{A. Troelstra}

Department of Medical Microbiology,

University Medical Centre Utrecht,

P.O. Box 85500, 3508 GA Utrecht, The Netherlands

C. H. van der Vaart

Department of Reproductive Medicine and Gynaecology,

University Medical Centre Utrecht,

P.O. Box 85500, 3508 GA Utrecht, The Netherlands
Keywords Bacterial colonisation - Genital prolapse · Infection · Polypropylene mesh

\section{Introduction}

In recent years, the use of synthetic mesh implants has rapidly become popular in pelvic organ prolapse surgery. The principal reason for its use is the observation that approximately one third of vaginal wall prolapses recur after classical midline fascia plication techniques [1]. Observational studies on monofilament polypropylene mesh implants have shown that the anatomical recurrence rate is lower after the use of mesh material as compared to classical prolapse repair without mesh [2-4].

However, the risks of infection and erosion when using mesh material are concerns currently being discussed. The properties of the mesh play an important role in lowering the infection risk. Currently, the so-called type 1 macroporous monofilament polypropylene mesh shows the lowest risk of infection and erosion and is therefore widely used. The use of tension-free type 1 polypropylene tapes in incontinence surgery has been shown not to be associated with a significant risk of mesh-related infections [5-7]. But, since the size of the meshes used for vaginal prolapse surgery and its positioning in the vagina differs substantially from the sub-urethral tapes, extrapolation of infection risk data on sub-urethral tapes cannot be made in advance. In theory, the risk of immediate or delayed infection can also be dependent on the sterility procedure during surgery. The objective of this study is to compare the intraoperative bacterial contamination of polypropylene mesh and clinical signs of infection between the hospital's routine and a more extensive intraoperative sterility procedure. 


\section{Materials and methods}

From December 2005 on, we use a collagen-coated transobturator polypropylene type 1 mesh (Avaulta ${ }^{\circledR}$, Bard, USA) for the repair of cystoceles and rectoceles $\geq$ stage 2 according to the POP-Q staging system [8]. Because of the theoretical protective effect of the collagen coating against a strong inflammatory response, and thereby possible erosion risk, we started to use this product. After gaining clinical experience with the implantation of 62 meshes, we started the study. All mesh kits consist of a core with four lateral arms. In anterior vaginal wall repair, we perform hydrodissection with $20-30 \mathrm{ml}$ of a saline/epinephrine $(1: 200,000)$ solution. After full thickness dissection of the vagina from the bladder, the paravesical space is entered and the mesh is placed with the use of four needle passages, outside-in, through the obturator foramen, according to the procedural guidelines of the Avaulta anterior product. In posterior repair, a full thickness dissection of the posterior vaginal wall is performed, after which the left and right pararectal spaces are entered and the sacrospinous ligament is identified. The mesh is placed through two skin incisions $3 \mathrm{~cm}$ lateral and $3 \mathrm{~cm}$ inferior to the anus. The curved needle is passed through the ischiorectal fossa parallel to the rectum towards the ischial spine on both sides to retrieve the two proximal arms of the mesh. Again, the procedural guidelines of the Avaulta posterior product are followed strictly. From September 2006, we started bacterial culturing of the meshes. We started with 37 consecutive women who received our routine surgical sterility policy, further referred to as method 1. From January 2007, we cultured meshes in 27 women who received additional sterility procedures, further described as method 2. Because of the lack of data on bacterial contamination of polypropylene mesh during vaginal surgery, we did not perform a sample-size calculation.

In method 1, no repeated disinfection of the operating area, no change of gloves or surgical instruments were performed during surgery. In method 2, a second disinfection of the surgical area with iodine, together with change of gloves and instruments, were performed just before the mesh was implanted.

Surgery was performed under general anaesthesia or spinal analgesia, according to patients' preference.

Before surgery, all women received a single dose of prophylactic antibiotics (intravenous cephazolin 1,000 mg and metronidazole $500 \mathrm{mg}$ ) at least $10 \mathrm{~min}$ before incision. The surgical area (vulva and vagina) was disinfected with povidone-iodine solution (Betadine, Meda Pharma, The Netherlands) and covered with sterile surgical drapes. This included a separate drape covering the anal region. The mesh was kept sterile in its package and was unpacked just before implantation. The time of mesh exposure between opening of the package and closing the vagina was measured in all women. After the mesh implantation, just before closing the vaginal wall, culture swabs (Transwab ${ }^{\circledR}$, MWE, UK) were taken from the central part of the mesh by diagonally crossing the mesh with the Transwab stick (from 10 to 4 o'clock and from 2 to 8 o'clock). Furthermore, the removed excessive materials of the mesh arms were sent to the microbiological laboratory for culturing.

Samples were transferred to microbial agar plates. These included blood agar, McConkey agar, Brucella blood agar with gentamycin for anaerobic bacteria and a mout agar for yeast (Becton Dickinson, Belgium). Samples were incubated for $48 \mathrm{~h}$ at $37^{\circ} \mathrm{C}$. All plates were evaluated semiquantitatively (expressed as colony-forming units per millilitre (CFU)) for the growth of Gram-negative, Grampositive, as well as anaerobic microorganisms and yeasts. Subsequently, colonies were determined on species level (Phoenix, Becton Dickinson) and susceptibility for cephazolin and metronidazole (Rosco, Taastrup, Denmark) was determined.

Postoperatively, an indwelling bladder catheter was used for 1 (posterior repair) or 3 (anterior repair) days. In all women baseline characteristics, intra- and postoperative complications were registered. Follow up was performed by scheduled visits to the outpatient clinic at 6 weeks and 6 months postoperatively. Mesh infection was evaluated by physical and vaginal examination, looking for signs of fever, redness, discharge, tenderness and swelling. If an infection was suspected, cultures of vagina, urine and blood were taken. Erosion was defined as any vaginal mesh exposition.

All data were analysed using SPSS 12.0.1. Comparisons between groups were made with Chi-square statistics for categorical and student $t$ tests for interval data. The significance level was set at a $p$ value of $<0.05$.

The study was approved by the local medical ethics committee.

\section{Results}

In Table 1 the characteristics of the both study populations are shown. A total of 68 implants, 39 Avaulta anterior and 29 Avaulta posterior, were implanted in 64 women. In one of the combined anterior and posterior procedures only the anterior mesh was cultured, leaving a total of 39 anterior and 28 posterior meshes that were cultured. No statistical significant differences were found between the two groups, except for postoperative fever. This occurred more often in women who had the extensive sterility procedure, but was of borderline significance $(p=0.07)$

In Table 2, the culture results of the central part of the mesh are shown. In a total of 56 implants $(83.6 \%)$ a 
Table 1 Patients characteristics

\begin{tabular}{lccc}
\hline Characteristics & Method $1(n=37)$ & Method $2(n=27)$ & $p$ \\
\hline Age $($ years, mean \pm SD) & $61(11)$ & $59(10)$ & 0.57 \\
BMI $\left(\mathrm{kg} / \mathrm{m}^{2}\right.$, mean $\left.\pm \mathrm{SD}\right)$ & $26(5)$ & $26(5)$ & 0.76 \\
Previous prolapse surgery, numbers (\%) & $20(54)$ & $17(63)$ & 0.68 \\
Anaesthesia, numbers (\%) & & & \\
General & $18(49)$ & $14(52)$ & 0.64 \\
Regional & $19(51)$ & & \\
Surgery, numbers $(\%)$ & & $14(52)$ & $12(44)$ \\
Anterior repair & $21(57)$ & $1(4)$ & 0.59 \\
Posterior repair & $13(35)$ & $75( \pm 44)$ & 0.27 \\
Combined repair & $3(8)$ & $42( \pm 12)$ & 0.42 \\
Blood loss (ml, mean \pm SD) & $68( \pm 58)$ & $18( \pm 4)$ & 0.63 \\
Operating time (min, mean \pm SD) & $39( \pm 12)$ & & \\
Time of mesh exposure (mean \pm SD) & $20( \pm 9)$ & $0(0)$ & \\
Complications, numbers $(\%)$ & $0(0)$ & $1(4)$ & 0.75 \\
Peroperative & $2(5)$ & $3(11)$ & 0.07 \\
Postoperative & $0(0)$ & $1(4)$ & 0.75 \\
Hematoma & $2(5)$ & & \\
Fever & & & \\
Mesh erosion & & & \\
\hline
\end{tabular}

positive culture was obtained from the central part. In nine implants, the cultured bacteria were potentially pathogenic but at a very low bacterial density $(<100 \mathrm{CFU})$. The bacterial density counted $100-1,000 \mathrm{CFU}$ in only two implants (3\%), both with non-pathogenic species.

In addition to the cultures from the central part of the mesh, the removed excess material of the arms from the mesh was also cultured. At least two arms were sent in for culture in all meshes except for one. A total of 207 mesh arms were cultured of which 197 (96\%) were colonised. This was not significantly different from the results of the central part of the mesh. The cultured species were equal to the central part of the mesh and 13 cultures $(6.3 \%)$ showed a bacterial density between 100 and 1,000 CFU, all coagulase-negative staphylococci and lactobacillus.

In Table 3, the differences in colonisation of the central part of the mesh between the two methods of handling of
Table 2 Culture results from the central part of the implant

Values are presented as numbers $(\%)$

\begin{tabular}{lllll}
\hline Bacterial species & None & $<10 \mathrm{CFU}$ & $10-100 \mathrm{CFU}$ & $>100-<1,000 \mathrm{CFU}$ \\
\hline Non-pathogenic species & & & & \\
Coagulase-negative Staphylococcus & $17(25.4)$ & $34(50.7)$ & $16(23.9)$ & - \\
Lactobacillus & $52(77.6)$ & $3(4.5)$ & $12(17.9)$ & - \\
Proprionibacteria & $55(82.1)$ & $7(10.4)$ & $5(7.5)$ & - \\
Corynebacterium & $55(82.1)$ & $8(11.9)$ & $3(4.5)$ & $1(1.5)$ \\
Group B Streptococcus (S. agalactiae) & $66(98.5)$ & $1(1.5)$ & - & - \\
Group C, D, G streptococci & $60(89.6)$ & $5(7.5)$ & $1(1.5)$ & $1(1.5)$ \\
Peptostreptococcus & $66(98.5)$ & $1(1.5)$ & - & - \\
Potential pathogenic species & & & & - \\
Staphylococcus aureus & $65(97)$ & $1(1.5)$ & $1(1.5)$ & - \\
Yeast & $67(100)$ & - & - & - \\
Escherichia coli & $64(95.5)$ & $3(4.5)$ & - & - \\
Klebsiella spp. & $67(100)$ & - & - & - \\
Bacteroides & $65(97)$ & $1(1.5)$ & $1(1.5)$ & - \\
Enterococcus & $66(98.5)$ & $1(1.5)$ & - & - \\
Proteus mirabilis & $66(98.5)$ & $1(1.5)$ & - & - \\
\hline
\end{tabular}


Table 3 Relationship between type of sterility procedures and bacterial growth on the mesh
Values are presented as numbers (\%)

\begin{tabular}{llll}
\hline Bacterial species & Method 1 (N=40) & Method 2 (N=27) & $p$ value \\
\hline Non-pathogenic species & & & \\
Coagulase-negative Staphylococcus & $31(77.5)$ & $19(70.4)$ & 0.13 \\
Lactobacillus & $8(20.0)$ & $7(25.9)$ & 0.16 \\
Proprionibacteria & $7(17.5)$ & $5(18.5)$ & 0.55 \\
Corynebacterium & $6(15.0)$ & $6(22.2)$ & 0.58 \\
Group B Streptococcus (S. agalactiae) & $1(2.5)$ & $0(0)$ & 0.41 \\
Group C, D, G streptococci & $2(5.0)$ & $5(19.5)$ & 0.25 \\
Peptostreptococcus & $1(2.5)$ & $0(0)$ & 0.41 \\
Potential pathogenic species & & & \\
Staphylococcus aureus & $1(2.5)$ & $1(3.7)$ & 0.34 \\
Yeast & - & - & - \\
Escherichia coli & $1(2.5)$ & $2(7.4)$ & 0.34 \\
Klebsiella spp. & - & - & - \\
Bacteroides & $0(0)$ & $2(7.4)$ & 0.22 \\
Enterococcus & $1(2.5)$ & $0(0)$ & 0.41 \\
Proteus mirabilis & $1(2.5)$ & $0(0)$ & 0.41 \\
\hline
\end{tabular}

the mesh are shown. No statistical significant differences were found. In addition, the two cultures from the central part of the mesh that showed a bacterial density between 100 and 1,000 CFU both occurred in the method 2 group.

In Table 4, the differences in colonisation of the central part of the mesh for the Avaulta anterior and Avaulta posterior are shown. The Avaulta anterior mesh was significantly more likely to have become colonised with coagulase-negative staphylococci as compared to the Avaulta posterior mesh. The opposite was true for the lactobacillus colonisation which appeared to occur more frequently in the posterior mesh, although this difference was of borderline statistical significance.
No postoperative fever was detected in any of the women of Method 1. In the method 2 group, three women had a body temperature above $38^{\circ} \mathrm{C}$ in the first 2 days after surgery. In one woman, it was measured once and no infectious origin could be detected. In two women, the fever resolved completely after oral antibiotics (amoxicillin/ clavunalate potassium). No clinical signs of infection of the mesh were found in these women. Blood, vaginal and urine cultures were negative. All women were seen at six weeks and six months after surgery and no signs infection of the surgical site were found.

In three women, a postoperative haematoma was diagnosed. These three women all developed an erosion/
Table 4 Relationship between site of implant and bacterial growth

Values are presented as numbers (\%)

${ }^{*} p<0.05$ with Pearson chisquare test

\begin{tabular}{llll}
\hline Bacterial species & Anterior $(N=39)$ & Posterior $(N=28)$ & $p$ value \\
\hline Non-pathogenic species & & & \\
Coagulase-negative Staphylococcus & $33(84.6)$ & $17(60.7)$ & $0.03^{*}$ \\
Lactobacillus & $5(12.8)$ & $3(11.1)$ & 0.09 \\
Proprionibacteria & $9(22.5)$ & $2(7.2)$ & 0.41 \\
Corynebacterium & $10(25.6)$ & $0(0)$ & 0.23 \\
Group B Streptococcus $($ S. agalactiae) & $1(2.6)$ & $1(3.6)$ & 0.39 \\
Group C, D, G streptococci & $6(15.5)$ & $1(3.7)$ & 0.45 \\
Peptostreptococcus & $0(0)$ & & 0.22 \\
Potential pathogenic species & & $1(3.6)$ & \\
Staphylococcus aureus & $1(2.6)$ & - & 0.35 \\
Yeast & - & $1(3.6)$ & - \\
Escherichia coli & $2(5.1)$ & - & 0.76 \\
Klebsiella spp. & - & $2(7.2)$ & - \\
Bacteroides & $0(0)$ & $1(3.6)$ & 0.24 \\
Enterococcus & $0(0)$ & $0(0)$ & 0.23 \\
Proteus mirabilis & $1(2.6)$ & & 0.39 \\
\hline
\end{tabular}


exposure, two cases in method 1 (anterior wall) and one case in method 2 (posterior wall). The erosions/exposures were all diagnosed within 4 months after surgery and located in the midline along the vaginal incision. The intraoperative cultures of the meshes of these women showed coagulase-negative staphylococci in two cases (both method 1) and no bacteria in one (method 2). The mesh at the erosion site was operatively excised, the vaginal wall closed again and these three women made an uneventful recovery.

\section{Discussion}

In this prospective observational study, we set out to detect the rate of bacterial colonisation of vaginally implanted collagen-coated polypropylene mesh, and if different sterility procedures during surgery were associated with differences in colonisation and clinical infection rate of vaginal implanted collagen-coated polypropylene mesh. We found that the colonisation rate is high, but the density of colonisation is far below the limit of what is known as clinical important contamination. All possible pathogenic microorganisms were susceptible to our antibiotic prophylaxis regime. We found no differences in colonisation, clinical infection and erosion/exposure rate between the two sterility procedures.

There are several potential drawbacks of our study that need to be discussed. First, our study is an observational study and therefore we cannot exclude the possibility that our two groups differed in characteristics. By using two consecutive cohorts, we tried to avoid potential indication bias. Secondly, we did not perform a sample-size calculation based on an expected difference between the two sterility procedures. If, with the results of our study in mind, we would have liked to detect a difference between a colonisation of $77.5 \%$ versus $70.4 \%$ (for coagulasenegative staphylococcus) between groups, we would need 500 women in each group to detect this difference with a power of 0.8 . For detecting the difference in $E$. coli detection (which was in favour to the standard sterile group), $2.5 \%$ versus $7.4 \%$, two groups of 381 women would be needed.

Thirdly, we did not compare a non-coated versus a coated polypropylene mesh, so any ideas on the possible protective value of the coating with respect to colonisation, remains purely speculative.

Our study shows that in the majority of women the vaginal collagen-coated polypropylene mesh becomes colonised during surgery. There are several issues of importance when discussing this finding. First, vaginal surgery is "clean-contaminated surgery" because the vagina is naturally colonised with bacteria. In our study, almost all cultured bacteria can be regarded as common vaginal microorganisms [9]. This finding, in combination with the finding that the bacterial density was low and all bacteria were susceptible to the antibiotic prophylaxis, is consistent with the fact that no clinical infections of the mesh occurred at 6 months follow up. Secondly, there is the issue of the definition of contamination. Culligan defined a bacterial contamination as any specimen culture that yielded $\geq 5000 \mathrm{CFU} / \mathrm{ml}$ [9]. Using this definition, none of the colonisations we found can be regarded as contamination, regardless of the sterility procedure used. Also, potential pathogenic microorganisms, like Staphylococcus aureus, Escherichia coli, Bacteroides species and yeast were seldom or not at all cultured and, if so, occurred in very low densities. Thirdly, the colonisation may have occurred because of an extensive exposure of the mesh to potential pathogens. Since we opened the mesh package just before implanting it and the mesh time exposure was only 18$20 \mathrm{~min}$ in all, we believe to have avoided excessive exposure as much as possible. The difference in colonisation between the anterior and posterior implants with respect to coagulase-negative staphylococcus might be explained from the handling of the mesh. In an anterior procedure, the mesh is reflected anteriorly after the first two proximal needle passages, bringing the mesh in contact with the vulva skin. After placement of two stay sutures, the implant is deflected away from the skin in order to pass the two distal needles. In case of the posterior procedure, the mesh is always placed over the perineum, which is covered by a sterile drape. So, contact with actual skin is minimal.

Another important finding of our study is the fact that repeated disinfection of the surgical area just before handling the mesh did not alter the colonisation rate and type of cultured microorganism. Hessami et al. showed that rapid recolonisation of the surgical area with vaginal bacteria occurred after standard iodine disinfection [10]. Thirty and $60 \mathrm{~min}$ after iodine disinfection, bacterial colonisation occurred in, respectively, $67 \%$ and $81 \%$ of cases. This rapid recolonisation supports our finding that after a mean mesh exposure time of $18 \mathrm{~min}$, we already found a similar bacterial colonisation rate between the two sterility techniques. Another study by Culligan et al. showed that during vaginal surgery, $52 \%$ of vaginal cultures were contaminated ( $>5,000 \mathrm{CFU} / \mathrm{Ml})$ after $30 \mathrm{~min}$ [9]. In addition, they showed that the number of positive cultures decreased sharply with an increasing time interval between the start of surgery (disinfection) and moment of vaginal culture. This indicates that the effect of antibiotic prophylaxis is likely to be of far greater importance then the disinfection procedure itself. They postulated that efforts should be undertaken to reduce contamination in the first 30-90 min of surgery. However, our data show that 
repeated disinfection of the surgical region did not diminish the colonisation rate or the bacterial density. Also, we found no statistical significant differences in colonisation rate or bacterial species between the cultures taken from the central part of the mesh and lateral arms. Apparently, pulling the arms through, from the vagina outwards, exposes the mesh arms to vaginal bacteria.

In our study, none of the cultures showed bacterial density $>1,000$ CFU. This is in sharp contrast to the $52 \%$ reported by Culligan. One reason might be that we used a combination of cephazolin and metronidazole as antibiotic prophylaxis, whereas they only used cephazolin. Unfortunately, data on the species cultured during their study are not given, so a more extensive comparison cannot be made. Another reason might be that we cultured the mesh itself and did not collect specimens from another part of the vagina. Finally, the collagen coating of the mesh could have been protective against early significant colonisation, but of course our study was not designed to test this hypothesis.

In our study, 64 women received a vaginal repair with the Avaulta mesh and no clinical infections were found during a prospective follow up of 6 months. This is consistent with literature that shows that mesh-related infections occur infrequently $(0-8 \%)$ in vaginal pelvic organ prolapse surgery $[5,11-14]$.

Nevertheless, when pathogenic bacteria are involved, like S. aureus and Bacteroides species, infections can be very serious $[14,15]$. Our antibiotic prophylaxis regime is designed to prevent serious colonisation with these pathogens.

In our study, three women had an erosion/exposure of the mesh during the follow-up period. The difference between erosion and exposure is currently subject of discussion. If one states that an exposure always occurs in the line of incision and an erosion can occur anywhere in the vagina, our three women had an exposure. These women had a postoperative hematoma that was not drained or evacuated. Since all exposures occurred in the line of incision, and intraoperative cultures of the removed mesh in these women were either negative or showed non-pathogenic bacteria in low densities, we believe that the delayed wound healing was the cause of this mesh exposure in our series.

If mesh erosion is found, it raises the question if colonisation of the mesh is a risk factor for the development of erosion, or that erosion exposes the mesh to vaginal bacteria to become colonised. In a recent study, bacteriological cultures of mesh material were taken from 16 women who had a removal of their vaginal mesh due to erosion or infection. In women with removal of the mesh due to an erosion, all cultures revealed bacteria quantities below $10^{3} / \mathrm{ml}[16]$. Whether or not these erosions were due to contamination during initial surgery remains unclear, but this seems highly unlikely in view of our data showing that low-grade colonisation occurs during surgery very frequently. If a causal relationship should exist, we would have expected much more problems of infection or erosion in our series.

At this stage, the use of a type 1 monofilament macroporous $(>75 \mu \mathrm{m})$ polypropylene mesh carries the lowest infection and erosion risk in any kind of reconstructive surgery [11]. However, long-term data on safety with respect to the risk of infection and erosion in vaginal surgery are still lacking. Infection of mesh in abdominal wall reconstruction may occur several years after surgery [17]. Several bacteria species (like S. aureus and E. coli) are capable of biofilm formation. Such a biofilm may protect bacteria to the host-defence mechanism and could be associated with infection at a later stage. However, its specific role in infection and erosion of synthetic mesh still has to be determined [17].

\section{Conclusion}

In conclusion, this study shows no additional effect of implementing additional sterility procedures during surgery on the bacterial contamination of mesh material or on clinical infection rate. The routinely performed sterility procedure together with a broad spectrum antibiotic prophylaxis appears to be safe in vaginal prolapse surgery with type-1 collagen-coated polypropylene mesh material.

Conflicts of interest The senior author (C.H. van der Vaart) is involved in training programmes for the use of the Bard Avaulta ${ }^{\circledR}$ system. This training programme is provided by the Bard Company, and the senior author receives a financial compensation for his efforts. None of the authors, or their relatives, is otherwise involved in the Bard Company in terms of being a consultant, stock holder, or having any kind of financial compensation.

Open Access This article is distributed under the terms of the Creative Commons Attribution Noncommercial License which permits any noncommercial use, distribution, and reproduction in any medium, provided the original author(s) and source are credited.

\section{References}

1. Olsen AL, Smith VJ, Bergstrom JO, Colling JC, Clark AL (1997) Epidemiology of surgically managed pelvic organ prolapse and urinary incontinence. Obstet Gynecol 89:501-506

2. de Tayrac R, Devoldere G, Renaudie J, Villard P, Guilbaud O, Eglin G (2007) Prolapse repair by vaginal route using a new protected low-weight polypropylene mesh: 1-year functional and anatomical outcome in a prospective multicentre study. Int Urogynecol J Pelvic Floor Dysfunct 18:251-256

3. Sand PK, Koduri S, Lobel RW, Winkler HA, Tomezsko J, Culligan PJ et al (2001) Prospective randomized trial of 
polyglactin 910 mesh to prevent recurrence of cystoceles and rectoceles. Am J Obstet Gynecol 184:1357-1364

4. Maher C, Baessler K (2006) Surgical management of anterior vaginal wall prolapse: an evidence based literature review. Int Urogynecol J Pelvic Floor Dysfunct 17:195-201

5. Cosson M (2004) Risk of infection and prostheses: time out or a red flag? J Gynecol Obstet Biol Reprod (Paris) 33:559-560

6. Schraffordt Koops SE, Bisseling TM, Heintz AP, Vervest HA (2005) Prospective analysis of complications of tension-free vaginal tape from The Netherlands Tension-free Vaginal Tape study. Am J Obstet Gynecol 193:45-52

7. Shah DK, Paul EM, Amukele S, Eisenberg ER, Badlani GH (2003) Broad based tension-free synthetic sling for stress urinary incontinence: 5-year outcome. J Urol 170:849-851

8. Bump RC, Mattiasson A, Bo K, Brubaker LP, DeLancey JO, Klarskov P et al (1996) The standardization of terminology of female pelvic organ prolapse and pelvic floor dysfunction. Am J Obstet Gynecol 175:10-17

9. Culligan P, Heit M, Blackwell L, Murphy M, Graham CA, Snyder J (2003) Bacterial colony counts during vaginal surgery. Infect Dis Obstet Gynecol 11:161-165

10. Hessami S, McKinney TB, Dhamotharan S, Snkar N, Hassan KA (2007) Recolonisation during vaginal surgery. Int Urogynecol J Pelvic Floor Dysfunct 18(Suppl I):S25-S106
11. Birch C (2005) The use of prosthetics in pelvic reconstructive surgery. Best Pract Res Clin Obstet Gynaecol 19:979-991

12. Falagas ME, Velakoulis S, Iavazzo C, Athanasiou S (2007) Meshrelated infections after pelvic organ prolapse repair surgery. Eur J Obstet Gynecol Reprod Biol 134:147-156

13. Deffieux X, de Tayrac R, Huel C, Bottero J, Gervaise A, Bonnet K et al (2007) Vaginal mesh erosion after transvaginal repair of cystocele using Gynemesh or Gynemesh-Soft in 138 women: a comparative study. Int Urogynecol J Pelvic Floor Dysfunct 18:73-79

14. Abdel-Fattah M, Ramsay I, West of Scotland Study Group (2008) Retrospective multicentre study of the minimally invasive mesh repair devices for pelvic organ prolapse. BJOG 115:22-30

15. Athanasiou S, Matthaiou DK, Falagas ME (2006) Vaginal mesh infection due to Bacteroides melaninogenicus: a case report of another emerging foreign body related infection. Scand J Infect Dis 38:1108-1110

16. Boulanger L, Boukerrou M, Rubod C, Collinet P, Fruchard A, Courcol RJ et al (2008) Bacteriological analysis of meshes removed for complications after surgical management of urinary incontinence or pelvic organ prolapse. Int Urogynecol J Pelvic Floor Dysfunct 19:827-831

17. Engelsman AF, van der Mei HC, Ploeg RJ, Busscher HJ (2007) The phenomenon of infection with abdominal wall reconstruction. Biomaterials 28:2314-2327 